\title{
Quality of life: modified triple-branched stent graft implantation versus frozen elephant trunk technique
}

\author{
zeng-rong luo ${ }^{1}$, Jia-Hui Li ${ }^{1}$, mirong tang ${ }^{2}$, Liang-Wan Chen ${ }^{1}$, and Liang-Liang Yan $^{1}$ \\ ${ }^{1}$ Affiliation not available \\ ${ }^{2}$ Union Hospital, FuJian Medical University
}

September 11, 2020

\begin{abstract}
Background: To compare the effects of modified triple-branched stent implantation and frozen elephant trunk technique on the quality of life (QoL) of acute Stanford Type A aortic dissection (AAAD) patients at different follow-up times. Methods: Data from 175 AAAD survivors was collected which were divided into two groups according to different surgical techniques: (group A): modified triple-branched stent graft implantation; (group B): frozen elephant trunk. The SF-36 were used to assess the QoL at discharge(AD), the third postoperative month (POM3), and the twelfth postoperative month (POM12). Results: (1)The total scores at each time of both groups showed lower than the normal level; Group A scored higher than group B at some time points in terms of some items, and some items at POM3 and POM12 scored higher than at discharge. Older patients underwent modified triple-branched stent graft implantation. (2) There were less patients with heavy self-perceived burden in group A than group B and patients with heavy self-perceived burden decreased over time. (3) Young postoperative AAD patients in group B with heavy self-perceived burden, acute renal failure, long LOS and blood loss $(>1000 \mathrm{~mL} / 24 \mathrm{~h})$ seemed to get a worse QoL. Conclusions: The impact on QoL of the modified triple-branched stent graft implantation technique seemed to be better than those of frozen elephant trunk surgery in some items.
\end{abstract}

\section{Hosted file}

renamed_90708\begin\{CJK\}\{UTF8\}\{gbsn\} - \end\{CJK\}\selectlanguage\{english\}JCS.docx available } at https://authorea.com/users/358218/articles/480601-quality-of-life-modified-triplebranched-stent-graft-implantation-versus-frozen-elephant-trunk-technique 


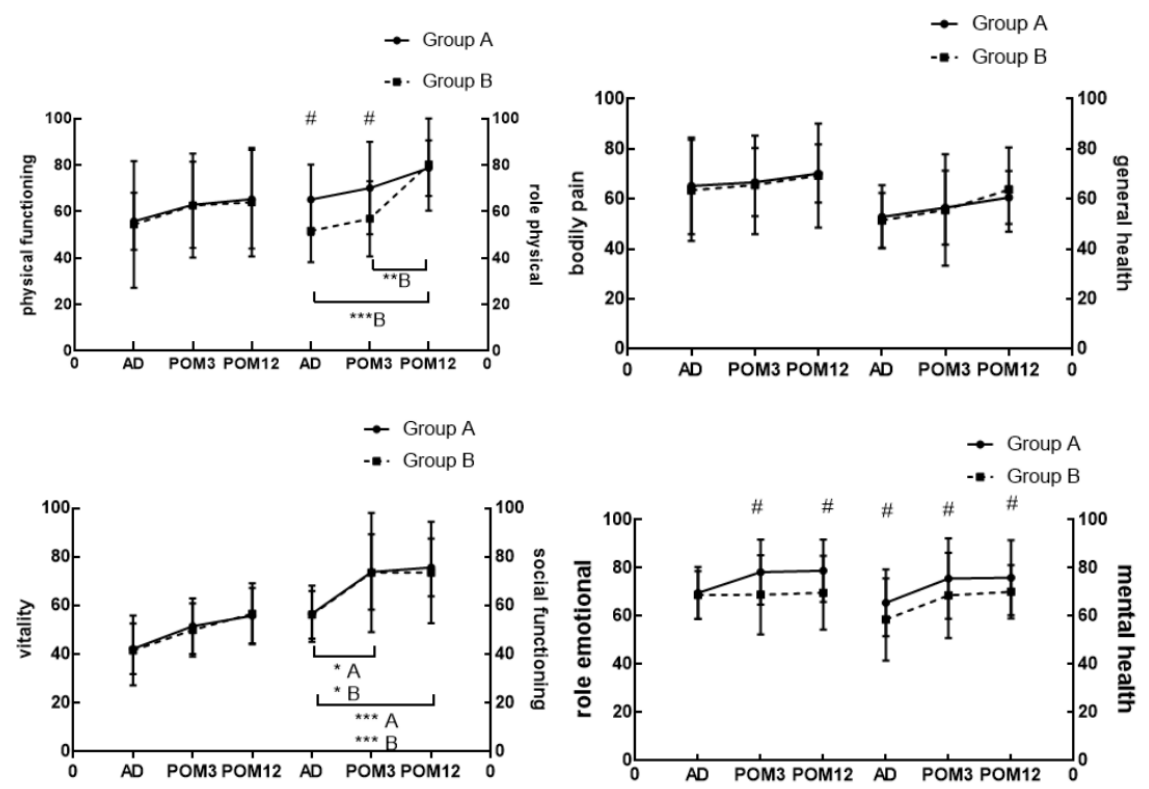

$\mathrm{AD}$ : at discharge, POM3: the third postoperative month, POM12: the twelfth postoperative month \#: Group A vs Group B ( $<<0.001)$, *A: AD vs POM3 of Group A $(P<0.001)$

$\star * \star A$ : $A D$ vs POM12 of Group $A(P<0.001), \quad * B: A D$ vs POM3 of Group $B(P<0.001)$

**B : POM3 vs POM12 of Group B $(P<0.001), \quad * * * B$ : AD vs POM12 of Group $B(P<0.001)$

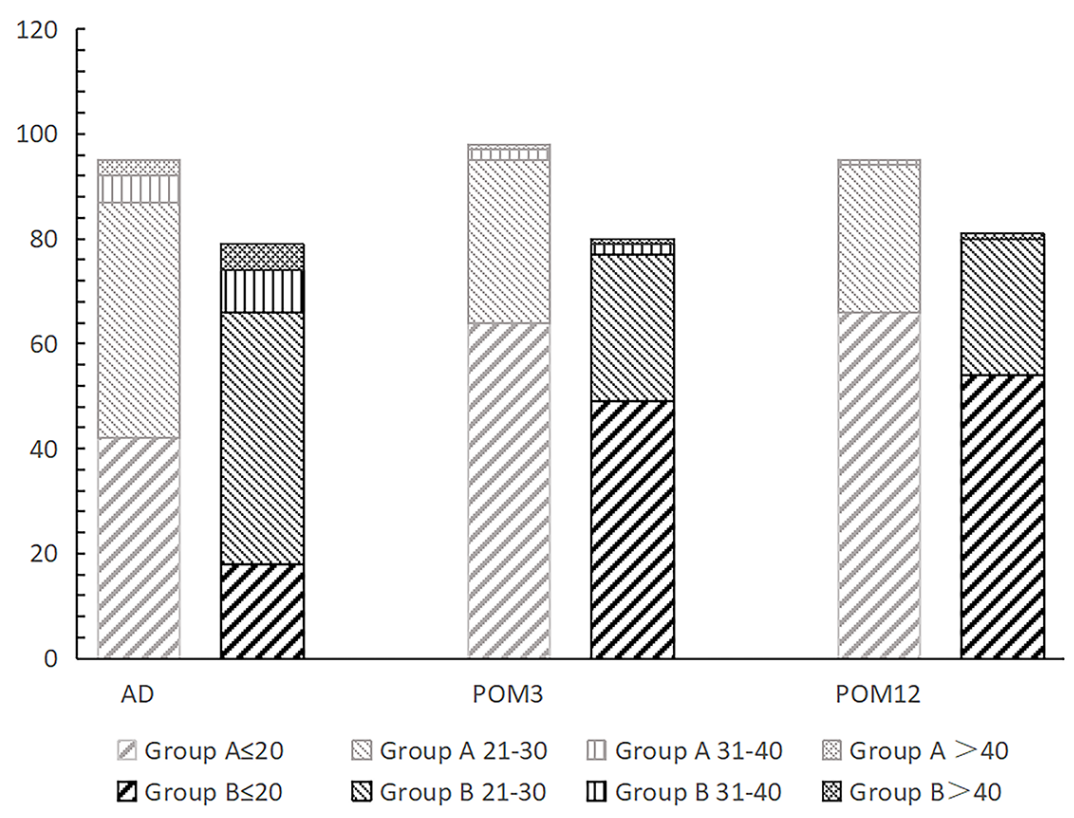

\section{Hosted file}

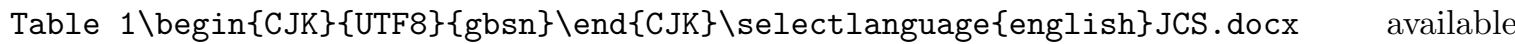
at https://authorea.com/users/358218/articles/480601-quality-of-life-modified-triple- 
branched-stent-graft-implantation-versus-frozen-elephant-trunk-technique

\section{Hosted file}

Table 2\begin } \{ \text { CJK\} } \{ \mathrm { UTF } 8 \} \{ \text { gbsn } \} \backslash \text { end } \{ \mathrm { CJK } \} \backslash \text { selectlanguage\{english\}JCS.docx available }

at https://authorea.com/users/358218/articles/480601-quality-of-life-modified-triplebranched-stent-graft-implantation-versus-frozen-elephant-trunk-technique

\section{Hosted file}

Table 3.docx available at https://authorea.com/users/358218/articles/480601-quality-of-lifemodified-triple-branched-stent-graft-implantation-versus-frozen-elephant-trunk-technique 